\title{
HYBRID MODELLING METHODOLOGY APPLIED TO MICROSTRUCTURAL EVOLUTION DURING HOT DEFORMATION OF ALUMINIUM ALLOYS
}

\author{
C.M. Sellars, M.F. Abbod, Q. Zhu and D.A.Linkens \\ Institute for Microstructural and Mechanical Process Engineering: The University of Sheffield, \\ Mappin Street, Sheffield S1 3JD, UK
}

Keywords: Aluminium alloys, thermomechanical processing, hybrid modelling, microstructure.

\begin{abstract}
This paper considers how data based neurofuzzy modelling techniques for the poorly understood relationships between changing process histories and the evolution of the internal state variables of dislocation density, subgrain size and subgrain boundary misorientation can be combined with physically-based models to investigate the effects of the internal state variables on the flow stress and recrystallisation behaviour. The model uses genetic algorithms to optimise the constants and is validated for data on a range of aluminium-magnesium alloys of both high and commercial purity. It is shown that this hybrid modelling methodology supported by a knowledge base offers a flexible way to develop the microstructrural modelling as more data and better understanding of the evolution of the internal state variables become available.
\end{abstract}

\section{Introduction}

Modelling of microstructure evolution of aluminium alloys during thermomechanical processing is one of the desirable methods to predict the properties of the material and to design the processing conditions accordingly. During thermomechanical processing such as forging, rolling or extrusion, the strain, strain rate, temperature and composition are the key variables that change from one material to another and from one type of processing to another. Changes in the deformation conditions affect the evolution of dislocation substructure and subsequent recrystallisation.

The recovery and recrystallisation of the deformed structure is an integral part of the thermomechanical process where annealing might occur wholly or partially between rolling passes during hot deformation. Quantitative relationships have been developed which relate the recrystallisation to flow stress which in turn is determined by the strain, strain rate and temperature of deformation [1]. Furthermore, different constitutive equations have been developed for the relationship between stress, strain, strain rate, and temperature based on hot plane strain compression tests using commercial purity aluminium, $\mathrm{Al}-1 \mathrm{Mn}$ and $\mathrm{Al}-1 \mathrm{Mg}$ alloys at temperature of 300,400 and $500^{\circ} \mathrm{C}$ and equivalent strain rates of $0.25,2.5$ and $25 \mathrm{~s}^{-1}$ to an equivalent strain of 2 [2].

The hybrid grey-box model which has been developed in this study is based on the internal state variables (such as dislocation density, subgrain size and misorientation between subgrains [3]), of the material being deformed. Physically-based mathematical equations have been used to describe the relationship between the internal states and flow stress and recrystallisation behaviour for aluminium alloys. The static recrystallisation behaviour is characterised by the kinetics, i.e. the time to $50 \%$ recrystallisation, the recrystallised grain size and the texture developed.

\section{Physically-Based Modelling}

Physically-based modelling of thermomechanical processing is carried out by considering the evolution of the internal state of the materials resulting from the externally applied deformation conditions of temperature $(T)$, strain rate $(\dot{\varepsilon})$ and strain $(\varepsilon)$. The internal state is defined in terms of the dislocation structures developed by work hardening and dynamic recovery in aluminium alloys. As illustrated in figure 1 , these are characterised by the dislocation density inside subgrains $\left(\rho_{\mathrm{i}}\right)$, the 
subgrain size or spacing between dislocation walls $(\delta)$ and the misorientation across subgrain boundaries or dislocation walls $(\theta)$.

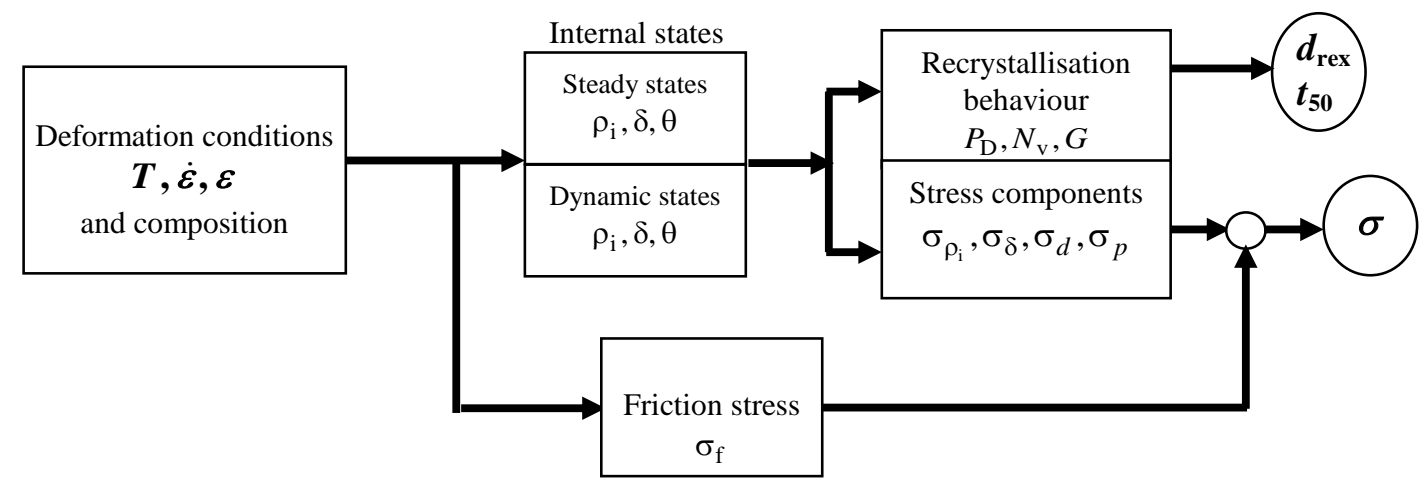

Figure 1: Block diagram of the hybrid model.

Industrial thermomechanical processing involves local regions undergoing changes of deformation conditions. Such changes involve a continuous change in strain rate and/or temperature and changes in strain path. A decrease in strain rate and change in straining direction has been found to have a significant effect on the evolution of deformation microstructure and subsequent recrystallisation behaviour [3-5]. The mechanism of the effects of change in strain rate or strain path on microstructural evolution is considered to be related to the different evolution of so-called geometrically necessary dislocations during monotonic and reversed deformation, and during constant or changing strain rates. The geometrically necessary dislocations, which are the local excess of the same sign, contribute to local lattice curvature. The local curvature $(1 / R)$ can be calculated from internal geometrically-necessary dislocation density $\left(\rho_{g}\right)$, subgrain size $(\delta)$ and misorientation across sub-boundaries $(\theta)$ as $1 / R=\rho_{\mathrm{g}} b+\theta / \delta$. During deformation after decreasing strain rate or change in straining direction, $1 / R$ initially remains constant but $\delta$ increases or $\theta$ decreases, so that $\rho_{g}$ increases. By considering these dynamic features of the evolution of dislocation density during thermomechanical processing, microstructural evolution has been successfully modelled $[3,5]$.

When the dislocation structure has been defined, the contributions to the flow stress arising from the internal dislocations $\left(\sigma_{\rho_{i}}\right)$, from the subgrain boundaries $\left(\sigma_{\delta}\right)$ and the grain boundaries $\left(\sigma_{\mathrm{d}}\right)$ and from second phase particles $\left(\sigma_{\mathrm{p}}\right)$, together with the contribution from the friction stress $\left(\sigma_{\mathrm{f}}\right)$, which is not microstructure sensitive, are computed [2]. The stored energy $\left(P_{\mathrm{D}}\right)$ is also computed from $\rho_{\mathrm{i}}, \delta$ and $\theta$ [3]. Nucleation of recrystallisation after plastic deformation takes place in different places within a deformed material. Nucleation at grain boundary surfaces is the dominant term for a large regime of deformation. As this strain regime covers most thermomechanical processing conditions, physically based equations are used for calculating nucleation density $\left(N_{\mathrm{V}}\right)$ and the average growth rate of recrystallisation grains $(\bar{G})$ using physically-based models. Then, recrystallisation characterised by the time of $50 \%$ recrystallisation $\left(t_{50}\right)$ and recrystallised grain size $\left(d_{\text {rex }}\right)$ can be calculated $[3,4]$. However, the current state of understanding of the physical metallurgy means that entirely "white box" models are not yet possible and some experimentally based empiricism must be involved in the models.

\section{Hybrid Modelling}

"Grey box" modelling techniques provide good modelling tools which combine different modelling approaches in one dynamics model [7]. Combinations of physically-based models and neurofuzzy models work very efficiently. While the physically-based model ensures that the results are physically sensible, the neurofuzzy model can be utilised to increase the accuracy of the results. One of the grey box modelling techniques is the "hybrid" semi-parametric model which utilises serial and parallel 
parametric and non-parametric models and gives advantages in compensating for sparse data, extrapolation improvements, uncertainty and the bias of the default model.

The ultimate aim of modelling the materials behaviour, however, is to be able to provide the tools that can be extended to all conditions and for any material. Currently, physically-based modelling is difficult to apply to extended conditions and materials. To extend the application requires the carrying out of a huge amount of experimental research, which is a very time-consuming process. Therefore, it is desirable to develop a modelling tool capable of being used for prediction under any extended deformation conditions and for any kind of material.

The hybrid modelling is carried out by combining the neurofuzzy models to predict the internal state variables with physically-based models to predict the final recrystallisation behaviour and flow stress from the internal state variables. Figure 2 shows the structure of the hybrid model. The heavy broken arrows show the "black box" neurofuzzy modelling steps to obtain the internal state variables. Also, the friction stress $\left(\sigma_{\mathrm{f}}\right)$ is at present difficult to calculate theoretically and hence is calculated using the neurofuzzy model based on experimental results. From the internal state variables, the heavy continuous arrows represent the "white box" steps using the physically based equations to calculate the final flow stress and recrystallisation behaviour. The architecture of the model is modular in form, so that individual modules can be modified as the knowledge base is developed by research, as indicated by the thin broken arrows. Also, since the model covers a wide range of temperature and strain rate for each individual composition, tuning the model is important in adjusting some of the parameters for scaling the internal state variables and calculating the individual internal stress components. A Genetic Algorithm (GA) is used to search for the best-fit parameters. These five parameters were optimised using a multi-objective function to minimise the error between the predicted stress and the measured stress. The GA was set to have a population size of 10 for 100 epochs with average multi-objective ranking [8].

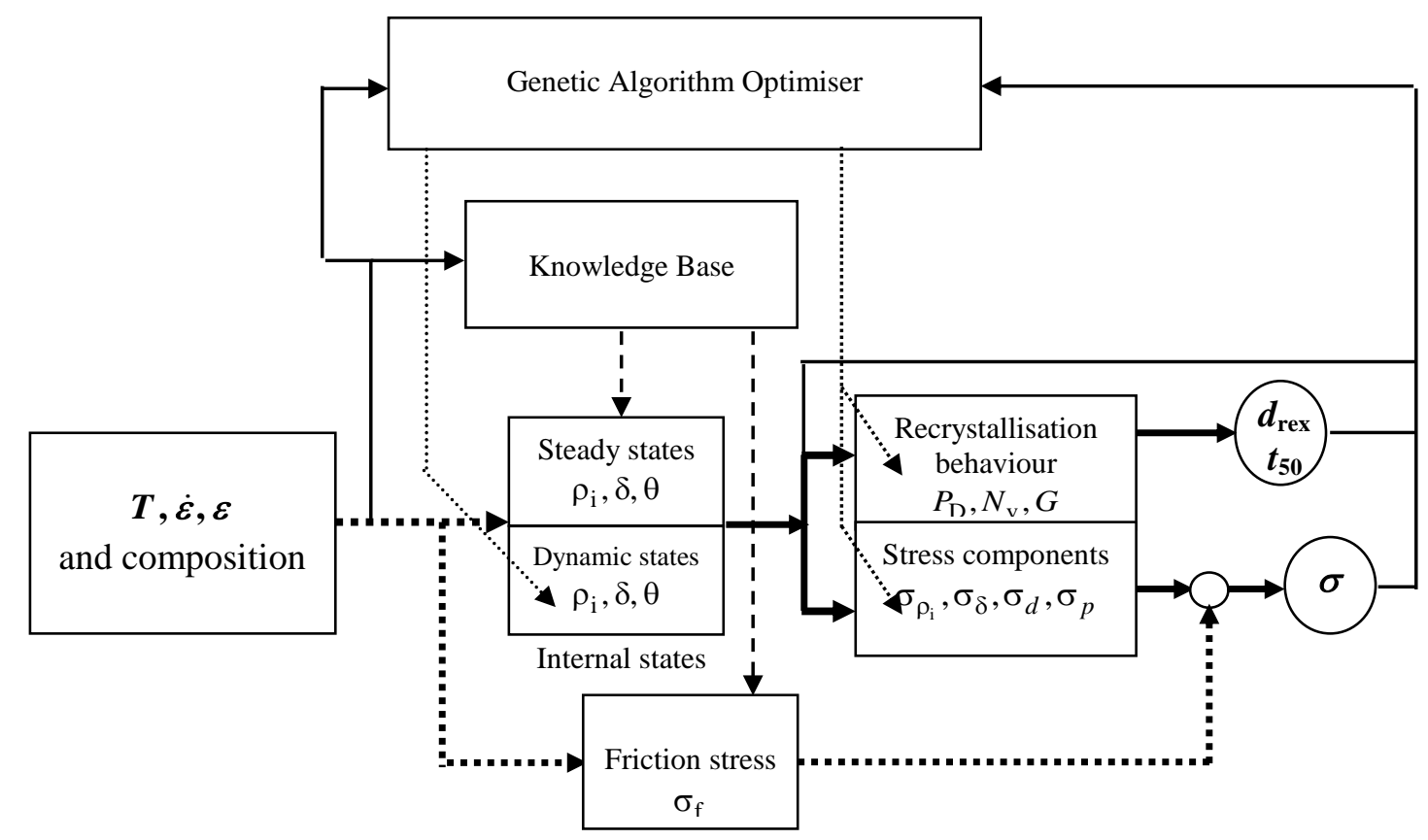

Figure 2: Block diagram of the hybrid modelling technique.

\section{Modelling Results}

Fig. 3 shows the predicted internal states against the experimental data for Al- $1 \% \mathrm{Mg}$ alloy under different deformation conditions. The "training" data show good prediction of internal dislocation density and subgrain size, figure 3(a) and (b), and no systematic errors for misorientation, figure 3(c). 
Further, the model has been used to predict the behaviour of the final properties of the material. Figure 4 shows the experimental data against the predicted recrystallisation behaviour and flow stress. Figure 4 (a), (b) and (c) again show good agreement for the "training" data on commercial purity $\mathrm{Al}-1 \% \mathrm{Mg}$.


Figure 3: Comparison of experimental [3-5] and hybrid-model results for (a) internal dislocation density, (b) subgrain size and (c) misorientation between subgrains in $\mathrm{Al}-1 \% \mathrm{Mg}$ after plane strain compression deformation at $385^{\circ} \mathrm{C}$ under constant $(\bullet 0.25 / \mathrm{s}$, $2.5 / \mathrm{s}, \quad \boldsymbol{\Delta} 25 / \mathrm{s})$ and transient deformation conditions.

\section{Model Validation}

The validation process and generalisation of the model to a wide range of deformation conditions and compositions comprises collecting data from the literature for different authors and different deformation conditions. The validation process of the grey-box model is based on the input-output relationship, which means the input deformation conditions, composition, and the output flow stress and the recrystallisation behaviour. The intermediate variables (internal state variables) are to be validated based on the output of the model using the physical equations. Thus, it is expected that for high purity aluminium the predicted flow stress based on the training data should be higher than the actual values. For higher magnesium commercial alloys such as AA5182, which contains $4.66 \% \mathrm{Mg}$, $0.31 \% \mathrm{Mn}$ and $0.21 \% \mathrm{Fe}$, the model under-predicts the flow stress, as shown in figure 4 (d) using 
results from two research teams $[9,10]$. However, the essential feature of this figure is that all the data for different deformation conditions fall closely about single line of slope 0.4 .

Further model prediction has been performed for different alloys based on the experimental results by different research groups and it has been shown that magnesium, manganese and iron are the most effective elements in strengthening aluminium alloys. Therefore, in order to develop a global model, the effects of the alloying elements have to be considered as the major inputs to the composition block. In designing experimental conditions for developing the whole model, the input parameters to be considered are the strain, strain rate, temperature and composition of $\mathrm{Mg}, \mathrm{Mn}$ and $\mathrm{Fe}$.

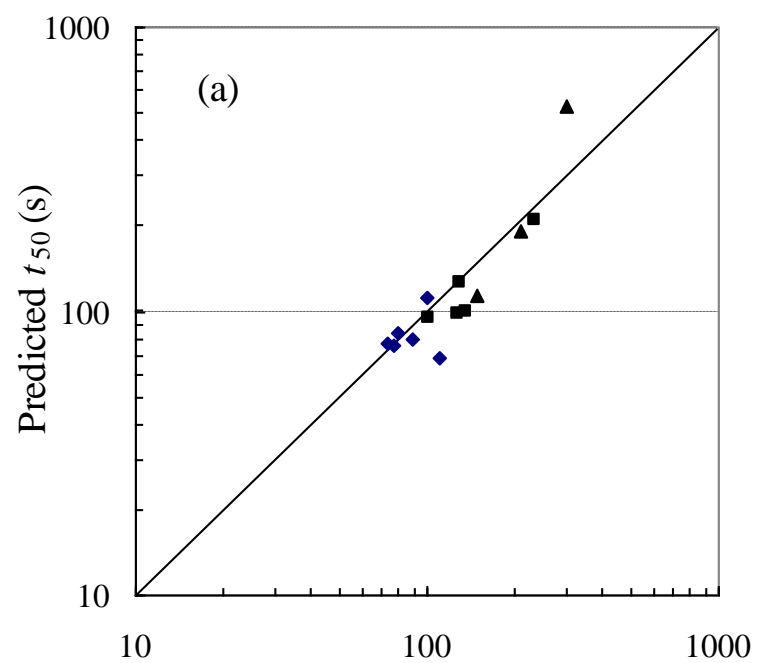

Experimental $t_{50}(\mathrm{~s})$

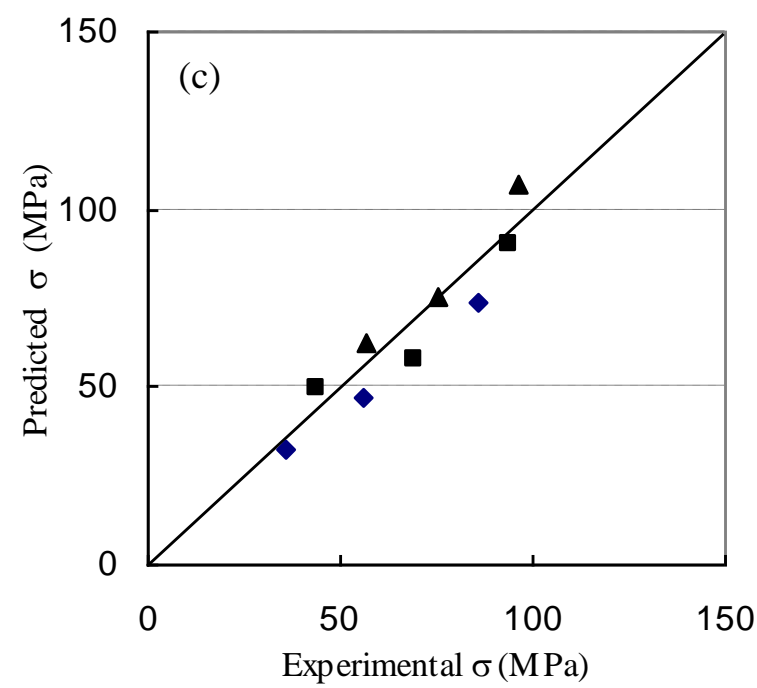

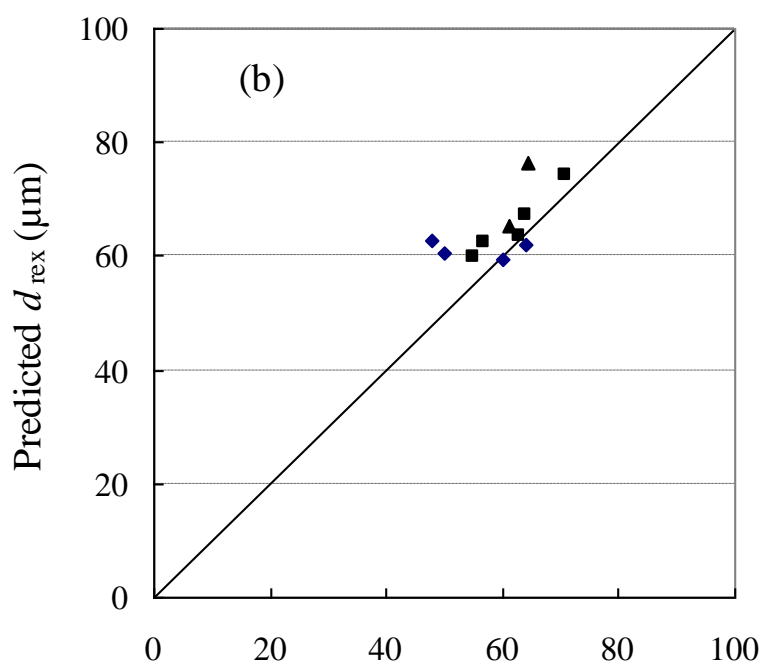

Experimental $d_{\text {rex }}(\mu \mathrm{m})$

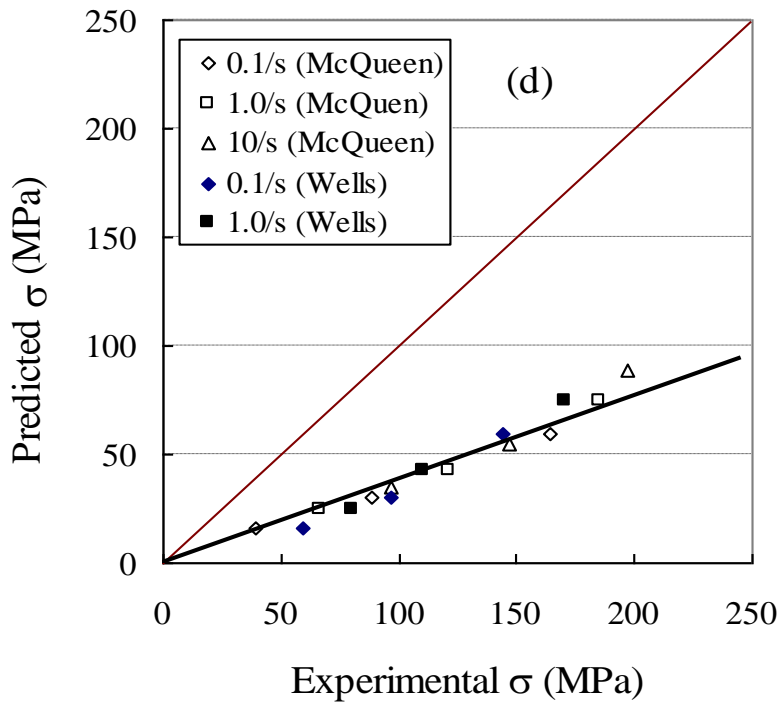

Figure 4: Comparison of experimental and hybrid-model results for (a) recrystallisation kinetics and (b) recrystallised grain size and (c) flow stress for commercial purity $\mathrm{Al}-1 \% \mathrm{Mg}$ after plane strain compression deformation at $385^{\circ} \mathrm{C}$ and under transient deformation conditions and after annealing at $400^{\circ} \mathrm{C}(\bullet 0.25 / \mathrm{s}, \boldsymbol{\square} 2.5 / \mathrm{s}, \boldsymbol{\Delta} 25 / \mathrm{s})$, and (d) the validation of the flow stress for AA5182 Al-4.5\% Mg alloy.

The slope of the lines predicted for $\mathrm{Al}-1 \% \mathrm{Mg}$ against experimental data is a good parameter to show the strengthening effect. Values smaller than unity represent under-prediction and greater than unity 
over-prediction of flow stress. Figure 5 shows the 3D surface for such slopes against the composition of magnesium and other impurities (mainly $\mathrm{Mn}$ and $\mathrm{Fe}$ ). As expected, the slope for the composition of $1 \% \mathrm{Mg}$ and $0.05 \%$ impurities is unity. With increasing magnesium content, the slope decreases. The slope is almost unaffected by impurity content greater than $0.05 \%$, because most of the $\mathrm{Fe}$ and $\mathrm{Mn}$ is out of solution as coarse particles. However, as the impurity content falls below the solubility limits, the slope and the effect of $\mathrm{Mg}$ increase rapidly.

The optimised hybrid model can be considered to be the best way to combine benefits of modelling for industry and for the research community. As industry is interested in the behaviour of the materials in terms of properties, the model can provide prediction capabilities. For the research community, however, the "white box" part of the model can be used in an inverse sense, i.e. the heavy arrows in figure 2 can be reversed in direction. Then, when only the input/output deformation conditions and material characteristics are available, the internal state variables can be predicted from the physically-based equations to give virtual "internal states". These can subsequently be used in designing the experimental conditions, which should reveal significant differences in microstructure, when undertaking time-consuming metallographic characterisation.

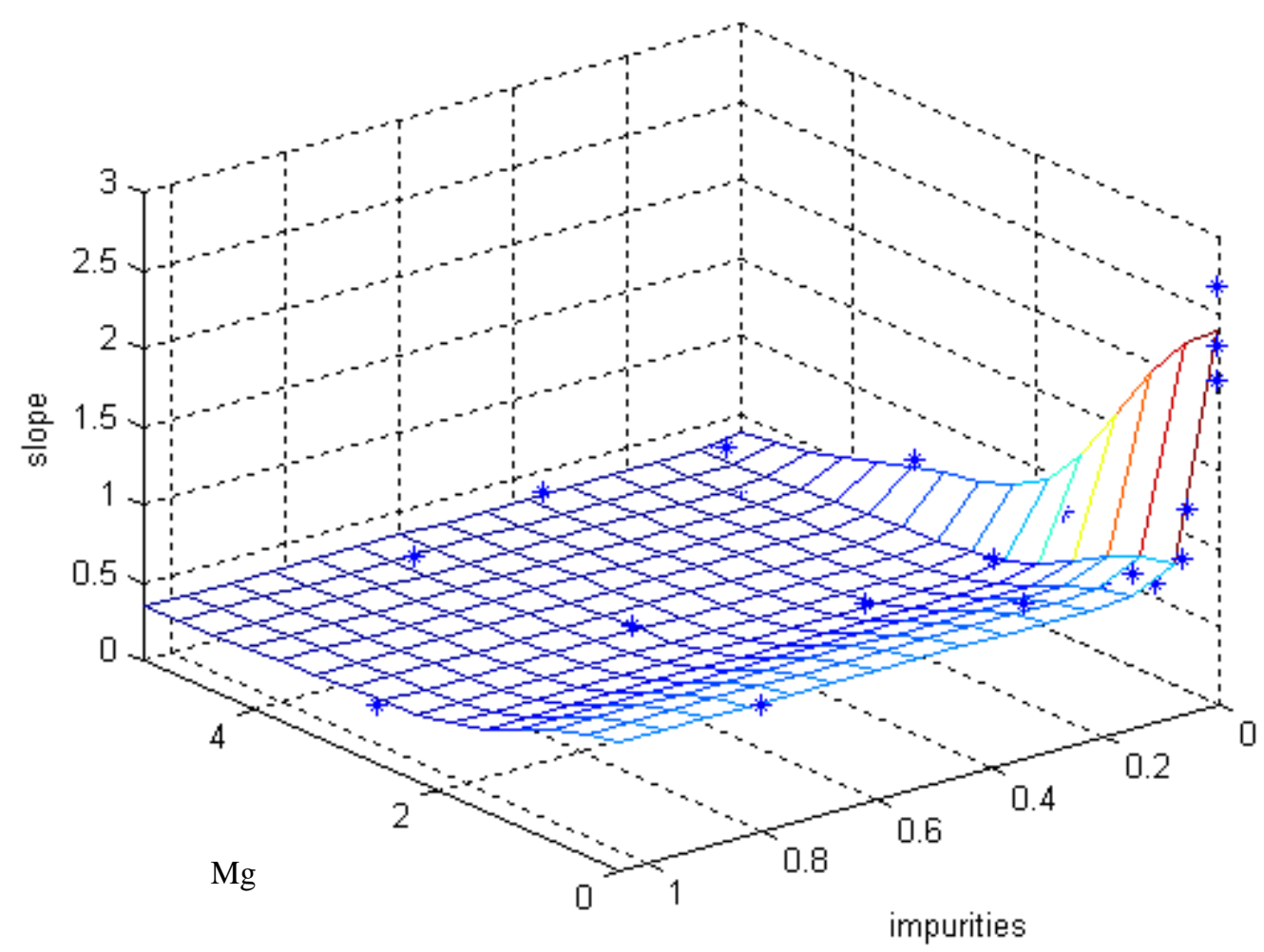

Figure 5: 3D surface showing the dependence on the impurity and $\mathrm{Mg}$ contents of the slope of the experimental versus predicted flow stress with respect to the $\mathrm{Al}-1 \% \mathrm{Mg}$ model.

\section{Knowledge Base}

The development of complex process models requires inputs from many resources of information, and must allow for upgrading as understanding and the knowledge base expands with future research. Because the developments take place over a relatively long time scale, it is highly desirable that the knowledge base used in the model is directly linked to the model so that it can be browsed and the model updated.

In developing the present hybrid model, a database has been compiled which contains all the information gathered during this research project. This provides an accessible source of information for the researchers in the future as well as giving insight into where the data came from, and how 
dense it is for certain process conditions, which reflects on the confidence in the prediction for particular conditions.

The model was developed in the Matlab environment. Matlab version 6.5 provides a dynamic link between MS Excel and its engine for exchanging data, as well as providing windows for text display and importing documents. This facility has been utilised to build the knowledge in Excel which is linked to the Simulink model developed in the Matlab environment. This dynamic link provides the facility to update the data points in Excel by either adding more data or upgrading the range of deformation conditions.

It was decided that the knowledge base should have three levels:

- First level: general information (author, deformation conditions, composition, etc)

- Second level: data points (tabulated into columns and also in graphical form).

- Third level: the source documents in PDF format.

An example of the second level type of information in the knowledge base is shown in figure 6 for the range of alloy composition and deformation conditions, represented in terms of the Zener Hollomon parameter $(Z)$, currently included in the validated hybrid model.

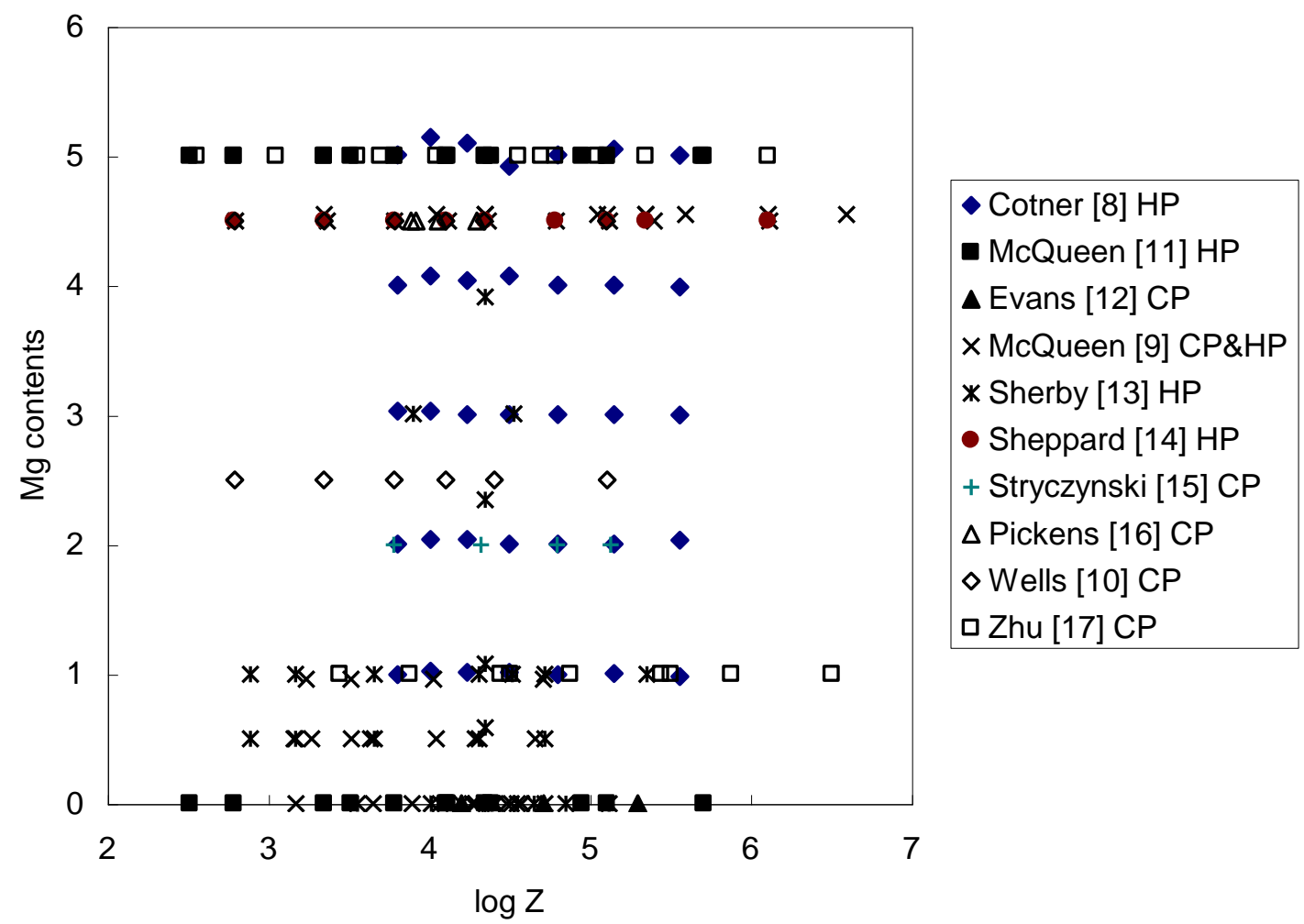

Figure 6: Data distribution for steady state flow stress for both high purity (HP) and commercial purity $(\mathrm{CP})$ aluminium alloys in the knowledge base.

\section{Conclusions}

The developed hybrid modelling technique combining the physically-based models and neurofuzzy models has been applied successfully to model the flow stress and evolution of internal state variables during thermomechanical processing and subsequent recrystallisation behaviour for different aluminium alloys.

The developed hybrid models have been validated against different experimental results under different deformation conditions from different research groups. The developed model can be used 
by both the research community and industry as it provides the internal state variables as well as the final material properties. Furthermore, the model can be used to predict the internal state variables which are difficult, expensive and time consuming to measure. The model has been linked to a knowledge base that describes the sources of the data as well as the operating range. This will give the model a "confidence band" to which the prediction is attached.

\section{Acknowledgements}

The authors are grateful to the UK Engineering and Physical Sciences Research Council for financial support for this research.

\section{References}

[1] P.L. Orsetti Rossi and C.M. Sellars: Acta Materialia, Vol. 45 (1997), 137-148.

[2] H. Shi, A. J. McLaren, C.M. Sellars, R. Shahani and R. Bolingbroke: Materials Science and Technology, Vol. 13 (1997), 210-216.

[3] Q. Zhu, H.R. Shercliff and C.M. Sellars: Int. Conf. on Thermomechanical Processing of Steels and Other Materials (THERMEC'97), eds T. Chandra and T Sakai, Wollongong/Australia, TMS (1997), pp. 2039-2046.

[4] C.M. Sellars and Q. Zhu: Materials Science and Engineering, Vol. A280 (2000), 1-7.

[5] G.J. Baxter, T. Furu, Q. Zhu, J.A. Whiteman and C.M. Sellars: Acta Materialia, Vol. 47 (1999), 2367-2376.

[6] H.U. Loffler, D. Gade, R. Doll, H. Muller, T. Peuker and G. Sorgel: IFAC Workshop, Future Trends in Automation in Mineral and Metal Processing, Finland, 22-24 August, (2000), pp.410-414.

[7] M.F. Abbod, D.A. Linkens and Q. Zhu: Materials Science and Technology, Vol. A333 (2002), 397-408.

[8] J.R. Cotner and W.J. McG. Tegart: Inst Metals, Vol. 97 (1969), 73-79.

[9] H.J. McQueen and Ryum: Scandinavian Journal of Metallurgy, Vol. 14 (1985), 183-194.

[10] M.A. Wells, D.J. Lloyd, I.V. Samarasekera, J.K. Brimacombe and E.B. Hawbolt: Metallurgical and Materials Transactions B. Vol. 29B (June 1998), 611-620.

[11] H.J. McQueen, W. Blum, Q, Zhu and V. Demuth: Advanced in Hot Deformation and Texture, eds T. R. Bieler and J. J. Jonas, Warrendale PA, TMS-AIME, 1994, pp.235-250.

[12] R.W. Evans and G.R. Dunstan: J. Inst. Et., Vol. 99 (1971), 4-14.

[13] O.L. Sherby and J. Wadsworth: Superplasticity in Aerospace, eds H.C. Heikkenen and T.R. McNelley, TMS-AIME, Warrendal PA (1988), pp. 3-28.

[14] T. Sheppard and M.G. Tutcher: Technology, Vol. 8 (1981), 319-327.

[15] A. Stryzczynski, Y. Esterin and H. Mecking: Creep and Fracture of Engineering Materials and Structures, eds B. Wilshire and D. Owen. Pineridge Press, Swansea, UK, (1984), pp. 115-130.

[16] J.R. Pickens, W. Precht and J.J. Mills: Proc of the 30th Sagamore Army Research Conference. eds G. Brugmann and V. Weiss, (1985), pp. 101-16.

[17] Q. Zhu, W. Blum and H.J. McQueen: Materials Science Forum, Vol. 217-222 (1996), pp. 1169-1174. 